November 4, 2022

\title{
Dual Higgs Mechanism for Quarks in Hadrons ${ }^{\star}$
}

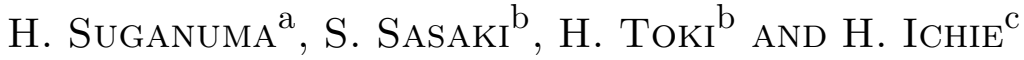 \\ a) The Institute of Physical and Chemical Research (RIKEN) \\ Hirosawa 2-1, Wako, Saitama 351-01, Japan \\ b) Research Center for Nuclear Physics (RCNP), Osaka University \\ Mihogaoka 10-1, Ibaraki, Osaka 56r, Japan \\ c) Department of Physics, Tokyo Metropolitan University \\ Minami-ohsawa, Hachiohji, Tokyo 192, Japan
}

\section{ABSTRACT}

* To appear in Proc. of the YITP Workshop on "From Hadronic Matter to Quark Matter: Evolving View of Hadronic Matter", YITP Kyoto Japan, November 1994, Prog. Theor. Phys. (Supplement). 
We study nonperturbative features of QCD using the dual Ginzburg-Landau (DGL) theory, where the color confinement is realized through the dual Higgs mechanism brought by QCD-monopole condensation. The linear confinement potential appears in the QCD-monopole condensed vacuum. We study the infrared screening effect to the confinement potential by the light-quark pair creation, and derive a compact formula for the screened quark potential. We study the dynamical chiralsymmetry breaking $(\mathrm{D} \chi \mathrm{SB})$ in the DGL theory by solving the Schwinger-Dyson equation. QCD-monopole condensation plays an essential role to $\mathrm{D} \chi \mathrm{SB}$. The QCD phase transition at finite temperature is studied using the effective potential formalism in the DGL theory. We find the reduction of QCD-monopole condensation and the string tension at high temperatures. The surface tension is calculated using the effective potential at the critical temperature. The DGL theory predicts a large mass reduction of glueballs near the critical temperature. We apply the DGL theory to the quark-gluon-plasma (QGP) physics in the ultrarelativistic heavy-ion collisions. We propose a new scenario of the QGP formation via the annihilation of color-electric flux tubes based on the attractive force between them. 


\section{Dual Higgs Mechanism for Color Confinement}

The quantum chromodynamics (QCD) has been believed as the fundamental theory of strong interactions [1-4]. Because of the asymptotic freedom of QCD, the ordinary perturbative technique is useful for the analysis of the high-energy hadron reactions [1-4]. However, QCD in the infrared region exhibits the nonperturbative features like the color confinement and the dynamical chiral-symmetry breaking $(\mathrm{D} \chi \mathrm{SB})$. In particular, the color confinement is extremely unique in the nonperturbative QCD, so that it is difficult to find similar phenomena in the other field of physics. The color confinement is characterized by the vanishing of the color dielectric constant, and squeezing of the color electric flux [4]. In this flux-tube picture, the string tension about $1 \mathrm{GeV} / \mathrm{fm}$ is one of the most important quantities on the color confinement [4]. On the other hand, D $\chi \mathrm{SB}$ belongs a wide category of the spontaneous symmetry breaking, and is characterized by the chiral condensate $\langle\bar{q} q\rangle$, the pion decay constant $f_{\pi}$ and the constituent quark mass. For the study of the $\mathrm{D} \chi \mathrm{SB}$, the Schwinger-Dyson (SD) approach [5,6] provides a useful and powerful tool in terms of the dynamical mass generation of light quarks. The QCD phase transition is also an interesting subject in the recent hadron physics. [4,7] It is expected that the quark-gluon-plasma (QGP) phase appears in the high-temperature system, which would be realized in the ultrarelativistic heavy-ion collisions or in the early universe. The QGP phase is characterized as the deconfinement and the chiral-symmetry restoration.

\subsection{Analogy Between Superconductors and QCD Vacuum}

About 20 years ago, Y. Nambu [8-10] proposed an interesting picture for the color confinement based on the analogy between the superconductor and the QCD vacuum. In the superconductor, magnetic field is excluded due to the Meissner effect, which is caused by Cooper-pair condensation. As the result, the magnetic

flux is squeezed like the Abrikosov vortex in the type II superconductor [11]. On the other hand, the color-electric flux is excluded in the QCD vacuum, and therefore 
the squeezed color-flux tube is formed between color sources. Thus, these two systems are quite similar, and can be regarded as the dual version each other [3]. This idea is based on the duality of the gauge theories, which was pointed out by P.A.M. Dirac more than 50 years ago.

In this analogy, the color confinement is brought by the dual Meissner effect originated from color-magnetic monopole condensation, which corresponds to Cooper-pair condensation in the superconductivity. As for the appearance of color-magnetic monopoles in QCD, 't Hooft [12] proposed an interesting idea of the abelian gauge fixing, which is defined by the diagonalization of a suitable gauge-dependent variable. In this gauge, QCD is reduced into an abelian gauge theory with magnetic monopoles, which will be called as QCD-monopoles in order to distinguish from GUT-monopoles. The QCD-monopoles appear from the hedgehog-like configuration corresponding to the nontrivial homotopy class on the nonabelian manifold, $\pi_{2}\left(\mathrm{SU}\left(N_{c}\right) / \mathrm{U}(1)^{N_{c}-1}\right)=Z_{\infty}^{N_{c}-1}$. Then, the abelian gauge fixing is expected to provide the basis of the analogy between the superconductor and the QCD vacuum.

We compare the dual Higgs mechanism in the QCD vacuum with the ordinary Higgs mechanism in the superconductor as shown in Fig.1. In the superconductor, there are two kinds of degrees of freedom, the gauge field (photon) and the matter field corresponding to the electron and the metallic lattice, whose interaction provides the Higgs mechanism through Cooper-pair condensation. On the other hand, there is only the gauge field in the pure gauge QCD, and therefore it seems difficult to find the analogous point between these two systems. However, in the abelian gauge, the diagonal part and the off-diagonal part of gluons play different roles. While the diagonal gluon behaves as the gauge field, the off-diagonal gluon behaves as the charged matter and provides QCD-monopoles. Condensation of QCD-monopoles leads to mass generation of the dual gauge field through the dual Higgs mechanism [13,14], which is the dual version of the Higgs mechanism. Thus, QCD can be regarded as the dual superconductor in the abelian gauge. 
In this framework, the nonperturbative QCD is mainly described by the abelian gauge theory with QCD-monopoles, which is called as the abelian dominance. As for the validity of this scheme, many recent studies based on the lattice gauge theory have supported QCD-monopole condensation and the abelian dominance in the maximal abelian gauge [15-18].

It is worth mentioning that the dual Higgs scheme predicts the existence of the dual gauge field $\vec{B}^{\mu} \equiv\left(B_{3}^{\mu}, B_{8}^{\mu}\right)$ and the QCD-monopole $\chi_{\alpha}(\alpha=1,2,3)$ as the relevant degrees of freedom related to the color confinement $[8,13,14]$. It can be proved that both $\vec{B}^{\mu}$ and $\chi_{\alpha}$ are color-singlet, so that they can be observed as physical states. Since $\vec{B}^{\mu}$ and $\chi_{\alpha}$ appear in the gluon sector of QCD, they are identified as glueballs, which are flavor-singlet. The dual gauge field $\vec{B}^{\mu}$ appears as a massive axial-vector particle. The QCD-monopole $\chi_{\alpha}$ appears as a massive scalar particle. These particles correspond to the weak vector boson and the Higgs scalar in the electro-weak unified theory.

In this paper, we study nonperturbative features of QCD using the dual GinzburgLandau theory $[13,14,19]$, which is an infrared effective theory of QCD based on the dual Higgs mechanism by QCD-monopole condensation. We study the color confinement, the infrared screening effect due to the $q-\bar{q}$ pair creation and the dynamical chiral-symmetry breaking $[13,20,21]$. We also study the QCD phase transition at finite temperature using the effective potential formalism in the DGL theory [22]. Finally, we apply the DGL theory to the quark-gluon-plasma physics in the ultrarelativistic heavy-ion collisions. 


\section{Dual Ginzburg-Landau Theory and Quark Potential}

\subsection{QCD-Monopole Condensation in DGL Theory}

The dual Ginzburg-Landau theory (DGL) theory $[13,14]$ is considered as an infrared effective theory of QCD in the abelian gauge, and is described by the diagonal gluon $\vec{A}^{\mu} \equiv\left(A_{3}^{\mu}, A_{8}^{\mu}\right)$, the dual gauge field $\vec{B}^{\mu} \equiv\left(B_{3}^{\mu}, B_{8}^{\mu}\right)$ and the QCDmonopole field $\chi_{\alpha}(\alpha=1,2,3)[13,14]$,

$$
\begin{aligned}
\mathcal{L}_{\mathrm{DGL}}= & -\frac{1}{2 n^{2}}[n \cdot(\partial \wedge \vec{A})]^{\nu}[n \cdot *(\partial \wedge \vec{B})]_{\nu}+\frac{1}{2 n^{2}}[n \cdot(\partial \wedge \vec{B})]^{\nu}[n \cdot *(\partial \wedge \vec{A})]_{\nu} \\
& -\frac{1}{2 n^{2}}[n \cdot(\partial \wedge \vec{A})]^{2}-\frac{1}{2 n^{2}}[n \cdot(\partial \wedge \vec{B})]^{2}+\bar{q}(i \not \partial-e \vec{H} \cdot \vec{A}-m) q \\
& +\sum_{\alpha=1}^{3}\left[\left|\left(i \partial_{\mu}-g \vec{\epsilon}_{\alpha} \cdot \vec{B}_{\mu}\right) \chi_{\alpha}\right|^{2}-\lambda\left(\left|\chi_{\alpha}\right|^{2}-v^{2}\right)^{2}\right]
\end{aligned}
$$

in the Zwanziger form [23], where the duality of the gauge theory becomes manifest. Here, $e$ is the gauge coupling constant, $g$ is the unit magnetic charge obeying the Dirac condition $e g=4 \pi$, and $\vec{\epsilon}_{\alpha}$ denotes the relative magnetic charge of the QCDmonopole field $\chi_{\alpha}[13,14]$. It should be noted that the magnetic charge $g \vec{\epsilon}_{\alpha}$ is pseudoscalar because of the extended Maxwell equation, $\nabla \cdot \mathbf{H}=\rho_{m}$. Hence, the dual gauge field $\vec{B}_{\mu}$ is axial-vector. In the absence of matter fields, one finds an exact dual relation between $\vec{A}_{\mu}$ and $\vec{B}_{\mu}$ in the field equation, $\partial \wedge \vec{B}={ }^{*}(\partial \wedge \vec{A})$.

In the DGL theory, the self-interaction of the QCD-monopole field $\chi_{\alpha}$ is introduced to realize QCD-monopole condensation. When QCD-monopoles are condensed, the dual Higgs mechanism occurs, and the dual gauge field $\vec{B}_{\mu}$ becomes massive, $m_{B}=\sqrt{3} g v$. The color-electric field is then excluded in the QCD vacuum through the dual Meissner effect, and is squeezed between color sources to form the hadron flux tube. The QCD-monopole also becomes massive as $m_{\chi}=2 \sqrt{\lambda} v$.

As for the symmetry of the DGL theory, there is the dual gauge symmetry $\left[\mathrm{U}(1)_{3} \times \mathrm{U}(1)_{8}\right]_{m}$ corresponding to the local phase invariance of the QCD-monopole 
field $\chi_{\alpha}[13,14]$ as well as the residual gauge symmetry $\left[\mathrm{U}(1)_{3} \times \mathrm{U}(1)_{8}\right]_{e}$ embedded in $\mathrm{SU}(3)_{c}$. The dual gauge symmetry leads to the conservation of the colormagnetic flux. In the QCD-monopole condensed vacuum, the dual gauge symmetry $\left[\mathrm{U}(1)_{3} \times \mathrm{U}(1)_{8}\right]_{m}$ is spontaneously broken due to mass generation of the dual gauge

field $\vec{B}_{\mu}$ through the dual Higgs mechanism. Hence, the color-magnetic flux is not conserved in the QCD-monopole condensed vacuum. On the other hand, the residual gauge symmetry $\left[\mathrm{U}(1)_{3} \times \mathrm{U}(1)_{8}\right]_{e}$ is never broken in this process [13].

In this framework, the Dirac condition $e g=4 \pi$ for the dual gauge coupling constant $g$ is naturally derived $[12,13]$ in the same way as in the Grand Unified Theory [2]. The DGL theory in the pure gauge is renormalizable, and is not asymptotically free on $g$ in view of the renormalization group. [See Eq.(5.2).] Hence, asymptotic freedom is expected for the gauge coupling constant $e$ owing to the Dirac condition. Thus, the DGL theory qualitatively shows asymptotic freedom on $e[13,20]$, which seems a desirable feature for an effective theory of QCD.

\subsection{Quark Confinement Potential}

We investigate the inter-quark potential in the quenched level using the DGL theory [13]. By integrating over $A_{\mu}$ and $B_{\mu}$ in the partition functional of the DGL theory, the current-current correlation $[13,14]$ is obtained as

$$
\mathcal{L}_{j}=-\frac{1}{2} \vec{j}_{\mu} D^{\mu \nu} \vec{j}_{\nu}
$$

with the nonperturbative gluon propagator,

$$
D_{\mu \nu}=\frac{1}{\partial^{2}}\left\{g_{\mu \nu}+\left(\alpha_{e}-1\right) \frac{\partial_{\mu} \partial_{\nu}}{\partial^{2}}\right\}-\frac{1}{\partial^{2}} \frac{m_{B}^{2}}{\partial^{2}+m_{B}^{2}} \frac{1}{(n \cdot \partial)^{2}} \epsilon_{\mu \alpha \beta}^{\lambda} \epsilon_{\lambda \nu \gamma \delta} n^{\alpha} n^{\gamma} \partial^{\beta} \partial^{\delta}
$$

in the Lorentz gauge. Putting a static quark with color charge $[3,13] \vec{Q}$ at $\mathbf{x}=\mathbf{r}$ and a static antiquark with color charge $-\vec{Q}$ at $\mathbf{x}=\mathbf{0}$, the quark current is written 
as $\vec{j}_{\mu}(x)=\vec{Q} g_{\mu 0}\left\{\delta^{3}(\mathbf{x}-\mathbf{r})-\delta^{3}(\mathbf{x}-\mathbf{0})\right\}$. We take $\mathbf{n} / / \mathbf{r}$, which is also used in the similar context of the dual string theory [8], because of the axial symmetry of the system and the energy minimum condition [13]. Otherwise, the energy of the system diverges.

We finally obtain the inter-quark potential including the Yukawa and the linear parts [13],

$$
V(r)=-\frac{\vec{Q}^{2}}{4 \pi} \cdot \frac{e^{-m_{B} r}}{r}+k r, \quad k=\frac{\vec{Q}^{2} m_{B}^{2}}{8 \pi} \ln \left(\frac{m_{B}^{2}+m_{\chi}^{2}}{m_{B}^{2}}\right)
$$

with $\vec{Q}^{2}=e^{2} / 3$ for quarks. Here, $m_{B}$ is the mass of the dual gauge field $\vec{B}_{\mu}$, whose inverse corresponds to the cylindrical radius of the flux tube. It should be noted that the expression of the string tension $k$ is quite similar to the energy per unit length of the Abrikosov vortex in the type-II superconductor [11].

We compare the static potential with the phenomenological one, for example, the Cornell potential [24]. We get a good agreement as shown in Fig.2 with the choice of $e=5.5, m_{B}=0.5 \mathrm{GeV}$ and $m_{\chi}=1.26 \mathrm{GeV}$ corresponding to $\lambda=25$ and $v=126 \mathrm{MeV}$, which provide $k=1.0 \mathrm{GeV} / \mathrm{fm}$ for the string tension and the radius

of the hadron flux as $m_{B}^{-1}=0.4 \mathrm{fm}$. Thus, the linear potential responsible for the quark confinement is reproduced in the DGL theory.

\section{Quark Pair Creation and Infrared Screening Effect}

In this chapter, we study the dynamical effect of light quarks and the infrared screening effect to the confinement potential. The dynamical effect of light quarks is important for the color confinement, because the linear quark potential is screened due to the $q-\bar{q}$ pair creation in the long distance [13]. In another words, a long hadron string can be cut through the light $q-\bar{q}$ pair creation. Hence, the static quark potential seems to be saturated in the infrared region. Such a tendency is observed in the lattice QCD with dynamical quarks [25]. 


\subsection{Quark-Pair Creation Rate in Hadron Flux Tubes}

We estimate the $q-\bar{q}$ pair creation rate in the color-electric field inside the hadron flux tube, which is formed between valence quarks. The Schwinger formula $[1,13,26,27]$ for the $q-\bar{q}$ pair creation rate $w$ is given by

$$
w=-\frac{N_{f}}{2 \pi^{2}} \int_{0}^{\infty} d p_{T} p_{T} \operatorname{tr}_{c}(e E) \ln \left\{1-e^{-\pi\left(p_{T}^{2}+M^{2}\right)(e E)^{-1}}\right\},
$$

where $M$ is the effective quark mass, and $E$ is the external color-electric field assumed as $E=\left(E_{3} T_{3}+E_{8} T_{8}\right)$. It should be noted that the $q-\bar{q}$ pair creation by the Schwinger mechanism is a nonperturbative effect in terms of the gauge coupling constant $e[13,26]$.

For the $q-\bar{q}$ pair creation in the hadron flux tube, one finds

$$
w=-\frac{N_{f}}{2 \pi^{2}} \int_{0}^{\infty} d p_{T} p_{T}\left[2 k \ln \left\{1-e^{-\pi\left(p_{T}^{2}+M^{2}\right) /(2 k)}\right\}+2 \cdot k \ln \left\{1-e^{-\pi\left(p_{T}^{2}+M^{2}\right) / k}\right\}\right] .
$$

The first term in the bracket corresponds to the creation of the $q-\bar{q}$ pair with the same color as the valence quark, and such a process contributes to the cut of the hadron flux tube [13]. In this case, the effective color charge of the created $q-\bar{q}$ pair becomes a half value due to the screening effect or the final-state interaction $[13,28]$, so that $2 k$ in the first term of Eq.(3.2) is reduced to $k$. On the other hand, the second term in Eq.(3.2) describes the creation of the $q-\bar{q}$ pair with different color from the valence quarks, so that this contribution is less important to the screening of the quark potential [13].

Thus, the $q-\bar{q}$ pair creation rate relevant to the screening effect is given by

$$
w_{\mathrm{sc}}=-\frac{N_{f}}{2 \pi^{2}} k \int_{0}^{\infty} d p_{T} p_{T} \ln \left\{1-e^{-\pi\left(p_{T}^{2}+M^{2}\right) / k}\right\} \equiv \int_{0}^{\infty} d p_{T} w\left(p_{T}\right),
$$

where $w\left(p_{T}\right)$ is the creation rate of the $q-\bar{q}$ pair with the transverse momentum $p_{T}$. The expectation value of the energy of the created $q-\bar{q}$ pair is estimated by 
$\left\langle 2 E_{q}\right\rangle \simeq \frac{1}{w_{\mathrm{sc}}} \int_{0}^{\infty} d p_{T} w\left(p_{T}\right) \cdot 2\left(p_{T}+M^{2}\right)^{1 / 2} \simeq 850 \mathrm{MeV}[13]$ for $k=1.0 \mathrm{GeV} / \mathrm{fm}$ and $M=350 \mathrm{MeV}$. Since the energy $\left\langle 2 E_{q}\right\rangle$ is supplied by the missing length of the hadronic string, the infrared screening length $R_{\mathrm{sc}}$ satisfies $k R_{\mathrm{sc}} \simeq\left\langle 2 E_{q}\right\rangle$. Hence, one obtains $R_{\mathrm{sc}} \simeq 1 \mathrm{fm}$ [13], which corresponds to a typical value of the hadron size.

\subsection{Infrared Screening Effect to Confinement Potential}

The hadronic string becomes unstable against the $q-\bar{q}$ pair creation when the distance between the valence quarks becomes larger than $R_{\mathrm{sc}}$. This means the vanishing of the strong correlation between the valence quarks in the infrared region, so that the corresponding infrared cutoff, $a \simeq R_{\mathrm{sc}}^{-1} \simeq 200 \mathrm{MeV}$, should be introduced to the system [13]. Taking account of such an infrared screening effect, we introduce the corresponding infrared cutoff $a$ to the nonperturbative gluon propagator $(2.3)$ by replacing $\frac{1}{(n \cdot k)^{2}} \rightarrow \frac{1}{(n \cdot k)^{2}+a^{2}}[13]$,

$$
D_{\mu \nu}^{s c}=-\frac{1}{k^{2}}\left\{g_{\mu \nu}+\left(\alpha_{e}-1\right) \frac{k_{\mu} k_{\nu}}{k^{2}}\right\}-\frac{1}{k^{2}} \frac{m_{B}^{2}}{k^{2}-m_{B}^{2}} \cdot \frac{\epsilon_{\mu \alpha \beta}^{\lambda} \epsilon_{\lambda \nu \gamma \delta} n^{\alpha} n^{\gamma} k^{\beta} k^{\delta}}{(n \cdot k)^{2}+a^{2}}
$$

because the non-local factor $\frac{1}{(n \cdot k)^{2}}$ provides the strong and long-range correlation as the origin of the confinement potential [13]. Here, this gluon propagator keeps the residual gauge symmetry. Such a disappearance of the infrared double pole in the gluon propagator in the DGL theory can be qualitatively shown by considering the polarization diagram of quarks.

Using the gluon propagator (3.4), we obtain a compact formula [13] for the quark potential including the infrared screening effect due to the $q-\bar{q}$ pair creation,

$$
V_{\mathrm{sc}}(r)=-\frac{\vec{Q}^{2}}{4 \pi} \cdot \frac{e^{-m_{B} r}}{r}+k \cdot \frac{1-e^{-a r}}{a}
$$

which exhibits the saturation for the longer distance than $a^{-1} \simeq 1 \mathrm{fm}$. This formula for the screened quark potential is used for the phenomenological analysis of the hadron decay [29], and seems consistent with the recent studies of the lattice QCD including light dynamical quarks [25]. 


\section{Dynamical Chiral-Symmetry Breaking}

We now study the dynamical chiral-symmetry breaking (D $\chi \mathrm{SB})$ in the dual Ginzburg-Landau (DGL) theory [13,21], considering the relation between the color confinement and $\mathrm{D} \chi \mathrm{SB}$, which is suggested by the lattice QCD with dynamical quarks [4]. We investigate $\mathrm{D} \chi \mathrm{SB}$ in terms of the dynamical quark-mass generation in the QCD-monopole condensed vacuum by using the Schwinger-Dyson (SD) equation for massless quarks [13,21],

$$
S_{q}^{-1}(p)=\not p+\int \frac{d^{4} k}{i(2 \pi)^{4}} \vec{Q}^{2} \gamma^{\mu} S_{q}(k) \gamma^{\nu} D_{\mu \nu}^{\mathrm{sc}}(k-p) .
$$

Here, we use the gluon propagator $D_{\mu \nu}^{\mathrm{sc}}$ including the nonperturbative effects on the color confinement and the infrared screening in the presence of light quarks [13]. The quark propagator $S_{q}(p)$ is assumed as $S_{q}(p)^{-1}=\not p-M\left(-p^{2}\right)+i \epsilon$.

Taking the trace and making the Wick rotation in the $k_{0}$-plane, we obtain the SD equation in the Euclidean metric,

$$
M\left(p^{2}\right)=\int \frac{d^{4} k}{(2 \pi)^{4}} \vec{Q}^{2} \frac{M\left(k^{2}\right)}{k^{2}+M^{2}\left(k^{2}\right)} D_{\mu}^{\mu \mathrm{sc}}(k-p),
$$

where the trace of the gluon propagator (3.4) is given as

$$
D_{\mu}^{\mu \mathrm{sc}}(k)=\frac{1}{(n \cdot k)^{2}+a^{2}} \cdot \frac{1}{k^{2}} \cdot \frac{2 m_{B}^{2}}{k^{2}+m_{B}^{2}}\left\{k^{2}-(n \cdot k)^{2}\right\}+\frac{3+\alpha_{e}}{k^{2}}
$$

in the Lorentz gauge. After performing the angular integration, we obtain the final expression for the SD equation [13],

$$
\begin{aligned}
M\left(p^{2}\right) & =\int_{0}^{\infty} \frac{d k^{2}}{16 \pi^{2}} \frac{\vec{Q}^{2} M\left(k^{2}\right)}{k^{2}+M^{2}\left(k^{2}\right)}\left(\frac{4 k^{2}}{k^{2}+p^{2}+m_{B}^{2}+\sqrt{\left(k^{2}+p^{2}+m_{B}^{2}\right)^{2}-4 k^{2} p^{2}}}\right. \\
& +\frac{\left(1+\alpha_{e}\right) k^{2}}{\max \left(k^{2}, p^{2}\right)}+\frac{1}{\pi p_{T}} \int_{-k}^{k} d k_{n} \frac{1}{\tilde{k}_{n}^{2}+a^{2}} \\
& \left.\times\left[\left(m_{B}^{2}-a^{2}\right) \ln \left\{\frac{\tilde{k}_{n}^{2}+\left(k_{T}+p_{T}\right)^{2}+m_{B}^{2}}{\tilde{k}_{n}^{2}+\left(k_{T}-p_{T}\right)^{2}+m_{B}^{2}}\right\}+a^{2} \ln \left\{\frac{\tilde{k}_{n}^{2}+\left(k_{T}+p_{T}\right)^{2}}{\tilde{k}_{n}^{2}+\left(k_{T}-p_{T}\right)^{2}}\right\}\right]\right)
\end{aligned}
$$


with $\tilde{k}_{n} \equiv k_{n}-p_{n}$ and $k_{T} \equiv\left(k^{2}-k_{n}^{2}\right)^{1 / 2}$. Here, one finds $\vec{Q}^{2}=e^{2} / 3$ for quarks. Since, the integrand in the SD equation is non-negative, the existence of the nontrivial solution can be expected.

In solving the SD equation, we use the Higashijima-Miransky approximation $[5,6]$ with a hybrid type of the running coupling constant,

$$
\tilde{e}=e\left(\max \left\{p^{2}, k^{2}\right\}\right), \quad e^{2}\left(p^{2}\right)=\frac{48 \pi^{2}\left(N_{c}+1\right)}{\left(11 N_{c}-2 N_{f}\right) \ln \left\{\left(p^{2}+p_{c}^{2}\right) / \Lambda_{\mathrm{QCD}}^{2}\right\}}
$$

Here, $p_{c}$ is defined as $p_{c} \equiv \Lambda_{\mathrm{QCD}} \exp \left[\frac{24 \pi^{2}}{e^{2}} \cdot \frac{N_{c}+1}{11 N_{c}-2 N_{f}}\right]$ with $e=e(0)$ [13]. This ansatz naturally connects to the asymptotic freedom of the running coupling at large momentum. The coupling constant at low energy, $e\left(p^{2} \sim 0\right) \simeq e$, controls the strength of the linear confinement potential.

We show in Fig. 3 the quark mass function $M\left(p^{2}\right)$ with $e=5.5$ and $a=80 \mathrm{MeV}$. The QCD scale parameter is set to a realistic value $\Lambda_{\mathrm{QCD}}=200 \mathrm{MeV}$ in the $\overline{\mathrm{MS}}$ scheme. In order to see the effect of QCD-monopole condensation, we vary the mass of the dual gauge field, $m_{B}$. There is no non-trivial solution for the case with small $m_{B}<300 \mathrm{MeV}$. A non-trivial solution is barely obtained at $m_{B}=300 \mathrm{MeV}$, and $M\left(p^{2}\right)$ increases rapidly with $m_{B}$ as shown in Fig.3. Thus, QCD-monopole condensation provides a crucial contribution to $\mathrm{D} \chi \mathrm{SB}[13]$.

We further examine the result for $M\left(p^{2}\right)$ as shown in Fig. 4 for $m_{B}=0.5 \mathrm{GeV}$, which was used for the argument of the confinement potential in chapter 2 . The quark mass function $M\left(p^{2}\right)$ in the space-like region is directly obtained from the SD equation. We extrapolate $M\left(p^{2}\right)$ into the time-like region using a polynomial function as a simulation of the analytic continuation. This curve does not satisfy the on-shell condition $M^{2}\left(p^{2}\right)+p^{2}=0$ ( $p_{\mu}$ : Euclidean momentum), and hence the quark propagator does not have a physical pole $[13,21]$. This may indicate the light-quark confinement.

We find that monopole condensation makes the slope of $M\left(p^{2}\right)$ around $p^{2} \simeq 0$ larger as can be seen by comparing the $m_{B}=0$ case with the $m_{B} \neq 0$ case. 
This tendency can be physically explained as follows. The strong confining force between $q$ and $\bar{q}$ appears due to QCD-monopole condensation, and this attractive force should promote $q-\bar{q}$ pair condensation [13,21]. Since such an effect of the confining force becomes stronger in the infrared region $p^{2} \simeq 0$, the corresponding dynamical mass generation of quarks is much enhanced there, which provides the large slope of $M\left(p^{2}\right)$ at $p^{2} \simeq 0$. Thus, the light-quark confinement or the absence of physical poles of quarks is achieved due to this modification of the quark propagator in the infrared region by the confinement effect $[13,20,21]$.

We also calculate the other quantities related to $\mathrm{D} \chi \mathrm{SB}$ from the solution of the SD equation. The constituent quark mass in the infrared region is found to be $M(0)=348 \mathrm{MeV}$. The quark condensate is obtained as $\langle\bar{q} q\rangle=-(229 \mathrm{MeV})^{3}$. The pion decay constant is also calculated as $f_{\pi}=83.6 \mathrm{MeV}$ using the Pagels-Stoker formula [30]. These values are to be compared with the standard values; $M(0)=350$ $\mathrm{MeV},\langle\bar{q} q\rangle=-(225 \pm 50 \mathrm{MeV})^{3}$ and $f_{\pi}=93 \mathrm{MeV}$. Thus, several relevant quantities on $\mathrm{D} \chi \mathrm{SB}$ can be reproduced in the DGL theory $[13,21]$.

\section{QCD Phase Transition at Finite Temperature}

\subsection{Effective Potential Formalism}

In this chapter, we study the change of the QCD vacuum at finite temperature using the dual Ginzburg-Landau (DGL) theory in terms of QCD-monopole condensation $[22,31]$. To concentrate on the confinement properties, we study at the quenched level [4], where the quark degrees of freedom are frozen. In this case, we can drop the quark term in the DGL Lagrangian and perform integration over the gauge field $A_{\mu}$. Hence, we obtain the partition functional as [22]

$$
Z[J]=\int \mathcal{D} \chi_{\alpha} \mathcal{D} \vec{B}_{\mu} \exp \left(i \int d^{4} x\left\{\mathcal{L}_{\mathrm{DGL}}-J \sum_{\alpha=1}^{3}\left|\chi_{\alpha}\right|^{2}\right\}\right)
$$


where $\mathcal{L}_{\text {DGL }}$ has a simple form,

$$
\mathcal{L}_{\mathrm{DGL}}=-\frac{1}{4}\left(\partial_{\mu} \vec{B}_{\nu}-\partial_{\nu} \vec{B}_{\mu}\right)^{2}+\sum_{\alpha=1}^{3}\left[\left|\left(i \partial_{\mu}-g \overrightarrow{\epsilon_{\alpha}} \cdot \vec{B}_{\mu}\right) \chi_{\alpha}\right|^{2}-\lambda\left(\left|\chi_{\alpha}\right|^{2}-v^{2}\right)^{2}\right]
$$

Here, we have introduced the quadratic source term $[22,32]$ instead of the linear source term, which is commonly used. As is well-known in the $\phi^{4}$ theory [2,7], the use of the linear source term leads to an imaginary mass of the scalar field $\chi_{\alpha}$ in the negative-curvature region of the classical potential, and therefore the effective action cannot be obtained there due to the appearance of "tachyons". In this respect, there is an extremely advanced point in the use of the quadratic source term [32], because the mass of the scalar field $\chi_{\alpha}$ is always real even in the negativecurvature region of the classical potential owing to the contribution of the source $J$ to the scalar mass. [See Eq.(5.4).] Then, one obtains the effective action for the whole region of the order parameter without any difficulty of the imaginary-mass problem. Since this method with the quadratic source term is quite general, it is convenient to formulate the non-convex effective potential in the $\phi^{4}$ theory, the linear $\sigma$ model or the Higgs sector in the unified theory [1-3].

The effective potential at finite temperature, which physically corresponds to the thermodynamical potential, is then obtained as [22]

$$
\begin{aligned}
V_{\text {eff }}(\bar{\chi} ; T)=3 \lambda\left(\bar{\chi}^{2}-v^{2}\right)^{2} & +3 \frac{T}{\pi^{2}} \int_{0}^{\infty} d k k^{2} \ln \left(1-e^{-\sqrt{k^{2}+m_{B}^{2}} / T}\right) \\
& +\frac{3}{2} \frac{T}{\pi^{2}} \int_{0}^{\infty} d k k^{2} \ln \left(1-e^{-\sqrt{k^{2}+m_{\chi}^{2}} / T}\right) .
\end{aligned}
$$

Here, the masses of the QCD-monopole and the dual gauge field depend on the QCD-monopole condensate $\bar{\chi}$,

$$
m_{\chi}^{2}(\bar{\chi})=2 \lambda\left(3 \bar{\chi}^{2}-v^{2}\right)+J(\bar{\chi})=4 \lambda \bar{\chi}^{2}, \quad m_{B}^{2}(\bar{\chi})=3 g^{2} \bar{\chi}^{2}
$$

We show in Fig.5 the effective potential $V_{\text {eff }}(\bar{\chi} ; T)$ as a function of the QCDmonopole condensate $\bar{\chi}$. The (local-)minimum point, $\bar{\chi}_{\text {phys }}(T)$, of $V_{\text {eff }}(\bar{\chi} ; T)$ cor- 
responds to the physical (meta-)stable vacuum state. At $T=0$, one minimum appears at a finite $\bar{\chi}$, which corresponds to the QCD-monopole condensed phase. As the temperature increases, the minimum moves toward a small $\bar{\chi}$ value, and the second minimum appears at $\bar{\chi}=0$ above the lower critical temperature $T_{\text {low }} \simeq 0.38 \mathrm{GeV}$, which is analytically obtained using the high-temperature expansion $[7,22,31]$,

$$
T_{\text {low }}=2 v \sqrt{\frac{6 \lambda}{2 \lambda+3 g^{2}}} .
$$

The potential values at the two minima become equal at the thermodynamical critical temperature $T_{c} \simeq 0.49 \mathrm{GeV}$. The trivial vacuum $(\bar{\chi}=0)$ becomes stable above $T_{c}$. Thus, this phase transition is of the first order.

Here, we consider the possibility of the temperature dependence on the parameters $(\lambda, v)$ in the DGL theory. The critical temperature, $T_{c}=0.49 \mathrm{GeV}$, seems much larger than the lattice QCD result, $T_{c} \simeq 0.2 \mathrm{GeV}[4]$. Here, the self-interaction of $\chi_{\alpha}$ is introduced phenomenologically in the DGL Lagrangian, and it would be reduced at high $T$ according to the asymptotic freedom behavior of QCD. Here, we use a simple ansatz for the $T$-dependence on $\lambda[22], \lambda(T) \equiv \lambda\left(1-\alpha T / T_{c}\right)$, where $\alpha$ is determined as $\alpha=0.96$ so as to reproduce $T_{c}=0.2 \mathrm{GeV}$. (We take $\lambda(T)=0$ for $T>T_{c} / \alpha$.) The qualitative behavior is the same as in the above argument with a constant $\lambda$. We find a first-order phase transition again. Then, a large reduction of the self-interaction among QCD monopoles is expected near the critical temperature $T_{c}: \lambda\left(T \simeq T_{c}\right) \simeq 1$ is considerably smaller than $\lambda(T=0)=25$. Such a large reduction of $\lambda(T)$ near $T_{c}$ may be checked using the lattice QCD.

When light dynamical quarks are included, the chiral-symmetry restoration is also expected in the DGL theory as well as the deconfinement phase transition at the critical temperature, because QCD-monopole condensation is essential for $\mathrm{D} \chi \mathrm{SB}$ as demonstrated in the previous chapter. 


\subsection{Glueball Mass, String Tension and Surface Tension}

We investigate the masses of the dual gauge field $\vec{B}_{\mu}$ and the QCD-monopole field $\tilde{\chi}_{\alpha}$ at finite temperatures. Here, $\vec{B}_{\mu}$ and $\tilde{\chi}_{\alpha}$ would appear as the color-singlet glueball field with $1^{+}$and $0^{+}$, respectively $[14,20]$. In Fig.6, we show the glueball masses $m_{B}(T)$ and $m_{\chi}(T)$ using variable $\lambda(T)$. It is worth mentioning that $m_{B}(T)$ and $m_{\chi}(T)$ drop down to $m_{B}, m_{\chi} \sim T_{c}(\simeq 0.2 \mathrm{GeV})$ from $m_{B}, m_{\chi} \sim 1 \mathrm{GeV}$ near the critical temperature $T_{c}$. In other words, the QCD phase transition occurs at the temperature satisfying $m_{B}, m_{\chi} \simeq T$. Thus, our result predicts a large reduction of the glueball masses, $m_{B}$ and $m_{\chi}$, near the critical temperature $T_{c}$.

This result would be natural because of the following argument. In general, the thermodynamical factor $1 /\left(e^{\omega_{n} / T} \pm 1\right)$ for the single-particle energy $\omega_{n}$ becomes relevant only for $\omega_{n} \lesssim T$. Hence, one may guess relatively high critical temperature $T_{c} \gtrsim 1 \mathrm{GeV}$ in the pure gauge QCD, because only heavy glueballs appear as the elementary excitations there. However, the lattice QCD shows $T_{c} \simeq 200 \mathrm{MeV} \ll$ $1 \mathrm{GeV}$. This discrepancy would be solved by a large reduction of the glueball mass near the critical temperature, as was demonstrated in the DGL theory [22]. Similar glueball-mass reduction is also suggested by the thermodynamical studies based on the lattice QCD data [33].

We investigate the string tension $k(T)$ at finite temperatures. The string tension $k(T)$ is obtained as shown in Fig.7 by using Eq.(2.4). In the case of variable $\lambda(T)$, the string tension $k(T)$ decreases rapidly with temperature, and $k(T)$ drops down to zero around $T_{c}=0.2 \mathrm{GeV}$. Hence, one expects a rapid change of the masses and the sizes of the quarkonia according to the large reduction of $k(T)$ at high temperatures. We plot also the lattice QCD data in the pure gauge [34] by black dots. One finds that the variable $\lambda(T)$ case reproduces the lattice QCD data.

We can also estimate the surface tension $\sigma$ between the confinement and deconfinement phases using the effective potential at $T_{c}$ in the DGL theory. We

show in Fig. 8 the effective potential $V_{\text {eff }}\left(\bar{\chi} ; T_{c}\right)$ for the variable $\lambda(T)$. There are two minima at $\bar{\chi}=0, \bar{\chi}_{c}$ in $V_{\text {eff }}\left(\bar{\chi} ; T_{c}\right)$, and we choose the origin of the energy as 
$V_{\text {eff }}\left(0 ; T_{c}\right)=V_{\text {eff }}\left(\bar{\chi}_{c} ; T_{c}\right)=0$ for simplicity. The mixed phase includes both the confinement phase $\left(\bar{\chi}=\bar{\chi}_{c}\right)$ and the deconfinement phase $(\bar{\chi}=0)$. Here, we set the boundary surface in the mixed phase on $x y$-plane $(z=0)$ in order to estimate the surface tension. In this case, the system depends on $z$-coordinate only, and the boundary condition is given as

$$
\bar{\chi}(z=-\infty)=0, \quad \bar{\chi}(z=\infty)=\bar{\chi}_{c} .
$$

The surface tension $\sigma$ in the DGL theory is estimated as

$$
\sigma \simeq \int_{-\infty}^{\infty} d z\left\{3\left(\frac{d \bar{\chi}(z)}{d z}\right)^{2}+V_{\mathrm{eff}}\left[\bar{\chi}(z) ; T_{c}\right]\right\}
$$

where $\bar{\chi}(z)$ satisfies the boundary condition (5.6).

The figure of $V_{\text {eff }}\left(\bar{\chi} ; T_{c}\right)\left(0 \leq \bar{\chi} \leq \bar{\chi}_{c}\right)$ is approximated as a sine curve,

$$
V_{\mathrm{eff}}\left(\bar{\chi} ; T_{c}\right) \simeq \frac{h}{2}\left\{1-\cos \left(2 \pi \bar{\chi} / \bar{\chi}_{c}\right)\right\} \quad\left(0 \leq \bar{\chi} \leq \bar{\chi}_{c}\right)
$$

with $h$ the "height" of $V_{\text {eff }}\left(\bar{\chi} ; T_{c}\right)$. Then, the field equation of $\bar{\chi}(z)$ can be solved analytically like the sine-Gordon equation [35],

$$
\bar{\chi}(z) \simeq \frac{2 \sqrt{6}}{3} \tan ^{-1} e^{z / \delta}, \quad \delta \equiv \frac{\sqrt{3}}{\pi} \bar{\chi}_{c} / \sqrt{h}
$$

where $\delta$ denotes the thickness of the boundary between the two phases. We obtain a simple formula for the surface tension $\sigma$,

$$
\sigma \simeq \frac{4 \sqrt{3}}{\pi} \sqrt{h} \bar{\chi}_{c}
$$

One finds $\bar{\chi}_{c} \simeq 0.49 \mathrm{fm}^{-1}$ and $h \simeq 0.026 \mathrm{fm}^{-4}$ from $V_{\text {eff }}\left(\bar{\chi} ; T_{c}\right)$ in Fig.8. Hence, the surface tension is estimated as $\sigma \simeq(112 \mathrm{MeV})^{3}$, and the thickness of the border between the two phases is $\delta \simeq 1.68 \mathrm{fm}$. Since the above estimation has been done in the quenched level, the obtained results are to be compared with the lattice QCD data in the quenched level, e.g. $\sigma^{1 / 3} \sim 60 \mathrm{MeV}[36]$. 


\section{Application to Quark-Gluon-Plasma Physics}

Finally, we apply the DGL theory to the quark-gluon-plasma (QGP) physics in ultrarelativistic heavy-ion collisions. In a modern picture of the QGP formation $[26,28,37]$, many color-electric flux tubes are formed between heavy ions immediately after the collision. In this pre-equilibrium stage, there occurs $q-\bar{q}$ pair creation violently inside tubes by the Schwinger mechanism [26,28]. During this process, the energy of the color-electric field turns into that of the stochastic kinetic motion of quarks (and gluons). The energy deposition and the thermalization thus occur. So far, many studies has been done to the properties of the QGP phase $[4,7,22]$ in the equilibrium stage, however, the pre-equilibrium stage is also important in terms of the QGP formation [26].

For the study of the QGP formation, the DGL theory would provide a useful method, because it describes the properties of the color-electric flux tube, which are important in the pre-equillibrium system just after the ultrarelativistic heavyion collisions. These would be lots of flux tubes overlapping in the central region between heavy ions, when the energy of the collision is enough high. Hence the interaction between the flux tubes is very important in this case, although dynamics of the flux tubes is neglected in most studies of the QGP formation.

There are several kinds of flux tubes in the QCD system. Each flux tube is

characterized by the color charge $\vec{Q}[3,13]$ at its one end. To classify sorts of the flux tube, we call the flux tube with a red quark $(R)$ at its one end as " $R-\bar{R}$ flux tube", and so on. In this case, the "direction" of the color-electric flux in the flux tube should be distinguished. For instance, $\bar{R}$ - $R$ flux tube is different from $R-\bar{R}$ flux tube in terms of the flux direction.

We study the interaction between two color-electric flux tubes using the DGL theory. The color-electric charges at one end of the flux tubes are denoted by $\vec{Q}_{1}$ and $\vec{Q}_{2}$. We idealize the system as two sufficiently long flux tubes, and neglect the effect of their ends. We denote by $d$ as the distance between the two flux tubes. For $d \gg m_{\chi}^{-1}$, the interaction energy per unit length in the two flux tube system 
is estimated as

$$
E_{\text {int }} \simeq \frac{8 \pi \vec{Q}_{1} \cdot \vec{Q}_{2}}{e^{2}} m_{B}^{2} K_{0}\left(m_{B} d\right)
$$

where $K_{0}(x)$ is the modified Bessel function. Here, we have used the similar calculation on the Abrikosov vortex in the type-II superconductor [11].

As shown in Fig.9, there are two interesting cases on the interaction between two color-electric flux tubes.

(a) For the same flux tubes with opposite flux direction (e.g. $R-\bar{R}$ and $\bar{R}-R$ ), one finds $\vec{Q}_{1}=-\vec{Q}_{2}$ i.e. $\vec{Q}_{1} \cdot \vec{Q}_{2}=-e^{2} / 3$, so that these flux tubes are attracted each other. It should be noted that they would be annihilated into dynamical gluons in this case.

(b) For the different flux tubes satisfying $\vec{Q}_{1} \cdot \vec{Q}_{2}<0$ (e.g. $R-\bar{R}$ and $B-\bar{B}$ ), one finds $\vec{Q}_{1} \cdot \vec{Q}_{2}=-e^{2} / 6$, so that these flux tubes are attractive. In this case, they would be unified into a single flux tube (similar to $G-\bar{G}$ flux tube).

Based on the above calculation, we propose a new scenario on the QGP formation via the annihilation of the color-electric flux tubes. When the flux tubes are sufficiently dense in the central region just after ultrarelativistic heavy-ion collisions, many flux tubes are annihilated or unified. During the annihilation process of the flux tubes, lots of dynamical gluons (and quarks) would be created. Thus, the energy of the flux tubes turns into that of the stochastic kinetic motion of gluons (and quarks). The thermalization is achieved through the stochastic gluon self-interaction, and finally the hot QGP would be created. Here, the gluon selfinteraction in QCD plays an essential role to the thermalization process, which is quite different from the photon system in QED.

In more realistic case, both the quark-pair creation and the flux-tube annihilation would take place at the same time. For instance, the flux tube breaking $[26,28,37]$ would occur before the flux tube annihilation for the dilute flux tube system. On the contrary, in case of the extremely high energy collisions, these would be lots of flux tubes overlapping in the central region between heavy ions, 
and therefore the flux tube annihilation should play the dominant role in the QGP formation. In any case, the DGL theory would provide a calculable method for dynamics of the color-electric flux tubes in the QGP formation.

\section{Summary and Concluding Remarks}

We have studied nonperturbative features of QCD using the dual GinzburgLandau (DGL) theory. The dual Higgs mechanism brought by QCD-monopole condensation leads to the color confinement in the DGL theory. The linear confinement potential has been derived in the QCD-monopole condensed vacuum.

We have studied the infrared screening effect to the confinement potential by the light-quark pair creation. By introducing the corresponding infrared cutoff, we have derived a compact formula for the screened quark potential.

We have studied the dynamical chiral-symmetry breaking (D $\chi \mathrm{SB})$ in the DGL theory by solving the Schwinger-Dyson equation. We have found that QCDmonopole condensation plays an essential role to $\mathrm{D} \chi \mathrm{SB}$. The quark propagator is largely modified by the confinement effect in the infrared region, which would leads to the absent of physical poles of quarks.

We have studied the QCD phase transition at finite temperature using the effective potential formalism in the DGL theory. Here, we have proposed the use of the quadratic source term as a general powerful method in the $\phi^{4}$-like theory. We have found the reduction of QCD-monopole condensation and the string tension at high temperatures. The surface tension has been calculated using the effective potential at the critical temperature. In particular, the DGL theory predicts a large mass reduction of glueballs, the QCD-monopole and the dual gauge field, near the critical temperature.

Finally, we have applied the DGL theory to the quark-gluon-plasma (QGP) physics in the ultrarelativistic heavy-ion collisions. Based on the attractive force between color-electric flux tubes, we have proposed a new scenario of the QGP formation via the annihilation of the flux tubes. 
One of the authors (H.S.) is supported by the Special Researchers' Basic Science Program at RIKEN.

\section{REFERENCES}

1. C. Itzykson and J. B. Zuber, "Quantum Field Theory", (McGraw-Hill, New York, 1985) 1.

2. T. P. Cheng and L. F. Li, "Gauge theory of elementary particle physics", (Clarendon press, Oxford, 1984) 1.

3. K. Huang, "Quarks, Leptons and Gauge Fields", World Scientific, Singapore (1982) 1 .

4. M. Creutz, "Quarks, gluons and lattices", (Cambridge press, 1983) 1.

H. J. Rothe, "Lattice Gauge Theories", (World Scientific, 1992) 1.

5. K. Higashijima, Phys. Rev. D29 (1984) 1228; Prog. Theor. Phys. Suppl. 104 (1991) 1.

6. V. A. Miransky, Sov. J . Nucl. Phys. 38(2) (1983) 280.

V. A. Miransky, "Dynamical Symmetry Breaking in Quantum Field Theories (World Scientific, 1993) 1.

7. J. I. Kapusta, "Finite-Temperature Field Theory", Cambridge University Press, Cambridge, 1988) 1.

8. Y. Nambu, Phys. Rev. D10 (1974) 4262.

9. G. 't Hooft, "High Energy Physics", ed. A. Zichichi (Editorice Compositori, Bologna, 1975).

10. S. Mandelstam, Phys. Rep. C23 (1976) 245.

11. E. M. Lifshitz and L. P. Pitaevsii, Vol.9 of Course of Theoretical Physics, "Statistical Physics, Part 2", (Pergamon press, Oxford, 1981) 1.

12. G. 't Hooft, Nucl. Phys. B190 (1981) 455. 
13. H. Suganuma, S. Sasaki and H. Toki, Nucl. Phys. B435 (1995) 207.

14. T. Suzuki, Prog. Theor. Phys. 80 (1988) 929 ; 81 (1989) 752. S. Maedan and T. Suzuki, Prog. Theor. Phys. 81 (1989) 229.

15. A. S. Kronfeld, M. L. Laursen, G. Schierholz and U. -J. Wiese, Phys. Lett. B198 (1987) 516.

A. S. Kronfeld, G. Schierholz and U. -J. Wiese, Nucl. Phys. B293 (1987) 461.

16. T. Suzuki and I. Yotsuyanagi, Phys. Rev. D42 (1990) 4257.

17. S. Hioki, S. Kitahara, S. Kiura, Y. Matsubara, O. Miyamura, S. Ohno and T. Suzuki, Phys. Lett. B272 (1991) 326.

18. H. Shiba and T. Suzuki, Nucl. Phys. B (Proc. Suppl.) 34 (1994) 182.

19. S. Maedan, Y. Matsubara and T. Suzuki, Prog. Theor. Phys. 84 (1990) 130. S. Kamizawa, Y. Matsubara, H. Shiba and T. Suzuki, Nucl. Phys. B389 (1993) 563.

20. H. Suganuma, S. Sasaki and H. Toki, Proc. of Int. Conf. on "Quark Confinement and Hadron Spectrum", Como, Italy, (World Scientific, 1994).

S. Sasaki, H. Suganuma and H. Toki, ibid.

H. Toki, H. Suganuma and S. Sasaki, Nucl. Phys. A577 (1994) 353c.

21. S. Sasaki, H. Suganuma and H. Toki, preprint, RIKEN-AF-NP-172 (1994).

22. H. Ichie, H. Suganuma and H. Toki, preprint, RIKEN-AF-NP-176 (1994).

23. D. Zwanziger, Phys. Rev. D3 (1971) 880.

24. W. Lucha, F. F. Schöberl and D. Gromes, Phys. Rep. 200, No.4 (1991) 127.

25. W. Sakuler, W. Bürger, M. Faber, H. Markum, M. Müller, P. De Forcrand, A. Nakamura and I. O. Stamatescu, Phys. Lett. B276 (1992) 155.

W. Bürger, M. Faber, H. Markum and M. Müller, Phys. Rev. D47 (1993) 3034 . 
26. H. Suganuma and T. Tatsumi, Phys. Lett. B269 (1991) 371.

H. Suganuma and T. Tatsumi, Prog. Theor. Phys. 90 (1993) 379.

27. H. Suganuma and T. Tatsumi, Ann. Phys. (N.Y.) 208 (1991) 470.

28. N. K. Glendenning and T. Matsui, Phys. Rev. D28 (1983) 2890; Phys. Lett. B141 (1984) 419.

29. K.-T. Chao, Y.-B. Ding and D.-H. Qin, Commun. Theor. Phys. 18 (1992) 321.

30. H. Pagels and S. Stoker, Phys. Rev. D20 (1979) 2947; D22 (1980) 2876.

31. H. Monden T. Suzuki and Y. Matsubara, Phys. lett. B 294 (1992) 100.

32. P. Ring and P. Schuck, "The Nuclear Many-Body Problem", (SpringerVerlag, New York, 1980) 1.

33. J. Engels, F. Karsch, H. Satz and I. Montvay, Phys. Lett. B102 (1981) 332.

34. M. Gao, Nucl. Phys. B9 (Proc. Suppl.) (1988) 368.

35. R. Rajaraman, "Solitons and Instantons", (North-Holland, Amsterdam, 1982) 1.

36. Y. Iwasaki, K. Kanaya and L. Karkkainen, Phys. Rev. D49 (1994) 3540.

37. G. Gatoff, A. K. Kerman and T. Matsui, Phys. Rev. D36 (1987) 114. 


\section{Figure Captions}

Fig.1. Comparison between the superconductor and the QCD vacuum in the abelian gauge. The off-diagonal gluon behaves as the charged matter field in the abelian gauge, and contributes to the appearance of QCD-monopoles. The dual Meissner effect is brought by QCD-monopole condensation, which is the dual version of the Meissner effect caused by Coopr-pair condensation in the superconductivity.

Fig.2. The static quark potential $V(r)$ in the dual Ginzburg-Landau theory. The dashed curve denotes the Cornell potential.

Fig.3. The dynamical quark mass $M\left(p^{2}\right)$ as a function of the Euclidean momentum squared $p^{2}$ for $m_{B}=300,400$ and $500 \mathrm{MeV}$.

Fig.4. The dynamical quark mass squared $M^{2}\left(p^{2}\right)$ as a function of $p^{2}$. The dotted straight line denotes the on-shell state.

Fig.5. The effective potentials at various temperatures as functions of the QCDmonopole condensate $\bar{\chi}$. The crosses denote their minima.

Fig.6. The glueball masses at finite temperatures : $m_{B}(T)$ and $m_{\chi}(T)$. A large reduction of these masses is found near the critical temperature. The phase transition occurs at the temperature satisfying $m_{B}, m_{\chi} \simeq T$, which is denoted by the dotted line.

Fig.7. The string tensions $k(T)$ as functions of the temperature $T$ for a constant $\lambda$ and a variable $\lambda(T)$. The lattice QCD results in the pure gauge in Ref.[34] are shown by black dots.

Fig.8. The effective potential $V_{\text {eff }}\left(\bar{\chi} ; T_{c}\right)$ at the critical temperature $T_{c}$. There are two minima at $\bar{\chi}=0, \bar{\chi}_{c}$ in $V_{\text {eff }}\left(\bar{\chi} ; T_{c}\right)$, and $h$ denotes the height of $V_{\text {eff }}\left(\bar{\chi} ; T_{c}\right)$. The dashed curve is an approximate sine one as Eq.(5.8).

Fig.9. The annihilation process of the color-electric flux tubes during the QGP formation in ultrarelativistic heavy-ion collisions. (a) The same flux tubes 
with opposite flux direction are attracted each other, and are annihilated

into dynamical gluons. (b) The different flux tubes (e.g. $R-\bar{R}$ and $B-\bar{B}$ ) are attractive, and are unified into a single flux tube. 